\title{
Periodic Lateralized Epileptiform Discharges in Fulminant Form of SSPE
}

\author{
Ajith Cherian, Sapna Sreedharan, Seetharam Raghavendra, Dinesh Nayak, \\ Ashalatha Radhakrishnan
}

Can. J. Neurol. Sci. 2009; 36: 524-526

\section{Case Report}

Subacute sclerosing panencephalitis (SSPE) is a slowly progressive, fatal inflammatory disease of the central nervous system developing as a sequel to early measles infection. The diagnosis is established by Dyken's criteria (Dyken, 1985) that include long-interval generalized periodic complexes (GPC) in the EEG as one of the criterion ${ }^{1}$. Atypical EEG patterns can be encountered in those with SSPE and should be part of the differential of PLEDs.

A 16-year-old boy presented with a two month history of deterioration in handwriting and brief involuntary jerks of the right upper limb occurring every 10 to 15 minutes which later spread to involve the right side of the face also. The frequency of these jerks progressively increased to one to two per minute. The jerks later involved the left hand also and appeared synchronously with the right sided jerks. These jerks were associated with frequent falls. He progressively became withdrawn, apathetic and finally developed motor aphasia with preserved comprehension about six weeks into the illness. He had no history of seizures. His vision and hearing was normal. He had not received measles vaccination and had suffered from measles at the age of seven years, which he probably acquired from his younger brother who had it.

On admission he was conscious and alert with motor aphasia. Optic fundi were normal. There was a minimal right arm drift but tendon jerks were symmetrical and plantar responses were flexor. Sensory and cerebellar examination was normal. The most notable finding was the presence of slow myoclonic jerks involving the right sided limbs every 20 to 30 seconds lasting one to two seconds each, with occasional synchronous left upper extremity jerks. Over the next 72 hours he developed a subtle right supranuclear facial paresis along with right hemiparesis with brisk tendon reflexes and an extensor right plantar response.

Investigations showed routine biochemical tests, hematological parameters, renal and liver functions, serum electrolytes and thyroid function tests to be normal. Magnetic resonance imaging (MRI) done outside six weeks into the illness showed peritrigonal white matter hyperintensities. Serial scalp EEGs showed evolution as described.

Electroencephalogram done 14 days prior to admission revealed long interval GPC lasting one second once every six to seven seconds with normal background activity (BGA) between the discharges (Figure 1A). These findings were typical of SSPE. A second EEG done 12 days after the first one, (two days prior to admission) revealed disappearance of the GPC and appearance of high amplitude periodic delta activity of $1.5 \mathrm{~Hz}$ over the left frontal region, which was repeated throughout the recording with mild slowing of the BGA compared to first EEG (Figure 1B). During his admission a repeat EEG was performed 11 days after the second one at the point when he had developed subtle right hemiparesis. It revealed left hemispheric PLEDs at $1.5-2 \mathrm{~Hz}$ frequency intermixed with high amplitude delta activity. The right hemispheric BGA consisted of 3 to $4 \mathrm{~Hz}$ slowing (Figure $1 \mathrm{C)}$.

An EEG repeated four days later showed disappearance of PLEDs replaced by rhythmic high amplitude 1 to $1.5 \mathrm{~Hz}$ delta activity over the left frontocentral and temporal regions, while the right hemispheric activity consisted of low amplitude mixed theta- delta activity (Figure 1D).

Since the occurrence of PLEDs usually indicates a fresh insult to the hemisphere, MRI was repeated which showed only the same peritrigonal hyperintensities without any fresh structural changes in the left hemisphere (Figure 2).

Cerebrospinal fluid analysis showed 5 cells $/ \mathrm{mm}^{3}$ that were all lymphocytes, sugar $3.4 \mathrm{mmol} / \mathrm{L}$ (blood sugar $4.6 \mathrm{mmol} / \mathrm{L}$ ), protein $330 \mathrm{mg} / \mathrm{L}$. Cerebrospinal fluid antimeasles antibody titre was positive in 1:625 dilutions. A diagnosis of SSPE was made and the patient was initiated on levamisole $50 \mathrm{mg}$ twice a week along with sodium valproate. However, he rapidly worsened over the next one month to become bed bound and requiring ventilatory support. He succumbed to his illness three months after the onset of symptoms.

\section{Discussion}

We report a case of SSPE, which fulfilled Dyken's criteria ${ }^{1}$ with an atypical clinical course resulting in early death and EEG evolution from initial classical GPC to left hemispheric PLEDs. The classical EEG pattern in SSPE is characterized by stereotyped bilaterally synchronous and symmetrical high amplitude delta waves repeating every 4-15 seconds and is disease specific. Several atypical changes have been described

\footnotetext{
From the Sree Chitra Tirunal Institute for Medical Sciences \& Technology, Trivandrum, Kerala, India.

Received September 11, 2008. Final Revisions Submitted March 27, 2009. Correspondence to: Ashalatha Radhakrishnan, Sree Chitra Tirunal Institute for Medical Sciences \& Technology, Trivandrum, Kerala, 695011, India
} 

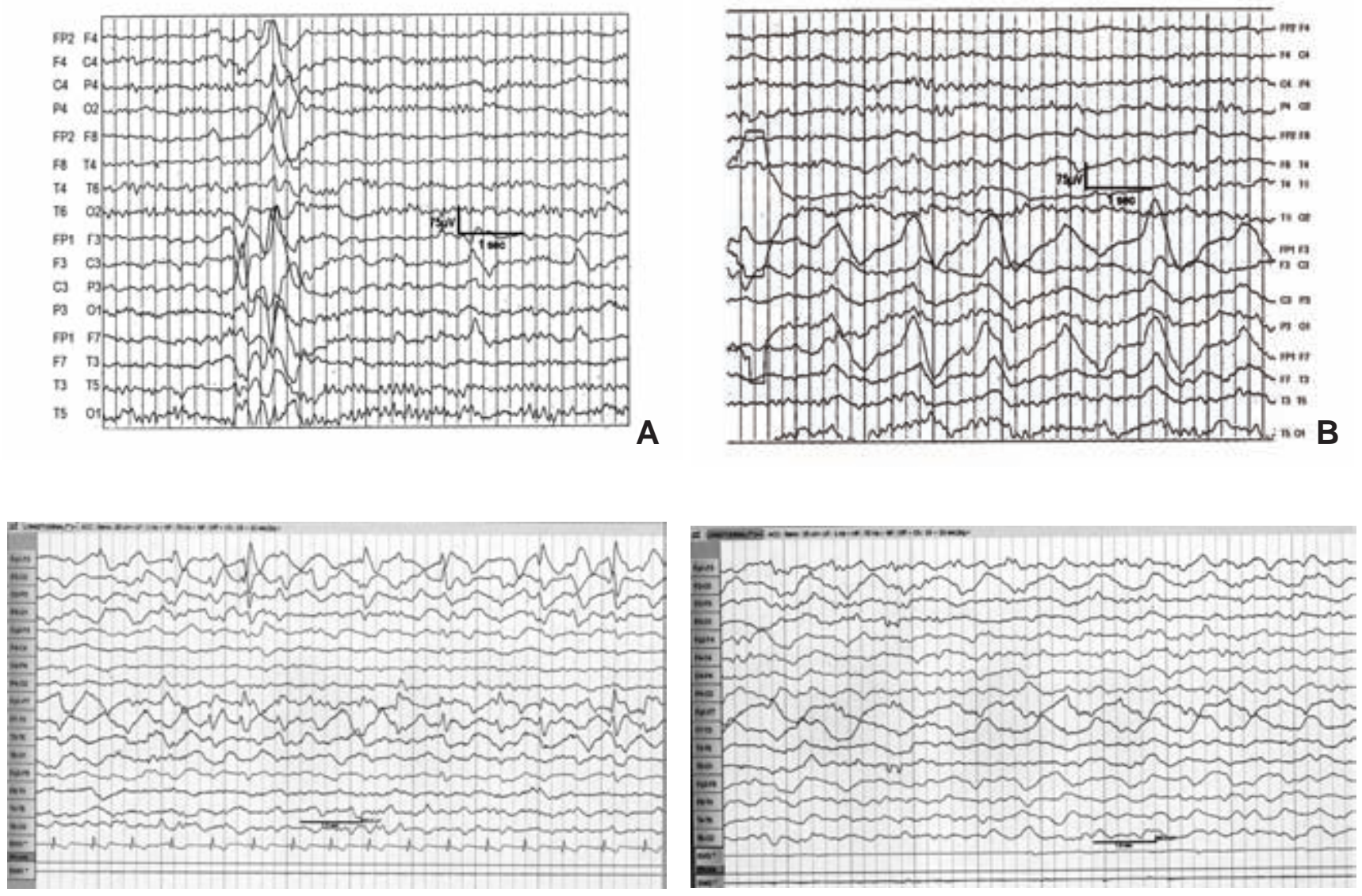

C

D

Figure 1: A: - Initial EEG of the patient showed generalized long interval high amplitude (250 - 300 microV) periodic complexes lasting about 1 second with well preserved BGA of 9.5-10 Hz with interburst interval of about 5-6 seconds. B: - Second EEG taken 12 days after the first one showed disappearance of the periodic complexes. It showed high amplitude (150 microV) quasirhythmic delta waves of frequency $1-1.5 \mathrm{~Hz}$ over the left frontal region. There was mild showing of the BGA(7.5-8 Hz) over the posterior head regions bilaterally. C: -Third EEG taken 23 days after the first one showed periodic short interval (1.5-2 sec) sharp waves (PLEDs) of 150 - 200 microV amplitude over the left front temporal region with high amplitude delta activity of 150 - 200 microV over the same hemisphere. The right hemisphere activity consisted of $3-4 \mathrm{~Hz}, 50-60 \mathrm{microV}$ theta -delta activity. D: EEG taken 27 days after the first showed rhythmic high amplitude $1-1.5 \mathrm{~Hz}$ delta activity at 200 microV over the left fronto centro temporal region intermixed with $7 \mathrm{~Hz}$ theta activity. Right hemisphere consisted of mixed theta delta activity. There was absence of PLEDs seen in Figure 1C.

which include asymmetric periodic complexes²; frontal rhythmic delta activity; electrodecremental periods following EEG complexes; diffuse sharp waves and sharp-and-slow-wave complexes over frontal regions; and focal abnormalities, such as sharp wave and sharp and slow wave foci. ${ }^{3}$

Combination of SSPE and PLEDs have been reported earlier, though rarely ${ }^{4-6}$. The electrical evolution in our patient was dissimilar and evolved from GPC to PLEDs over a period of three weeks but there were no clinical seizures through out the course of the illness as is expected in acute PLEDs ${ }^{7}$.

The clinical course in our patient was also atypical in that it was very rapid, with initial presentation as unilateral myoclonus along with weakness. Such an asymmetric presentation has been rarely reported earlier ${ }^{8}$. Ekmekci et al studied 22 patients with definite SSPE with atypical EEG abnormalities in relationship to the clinical features ${ }^{9}$. Atypical EEG changes were more often observed in later stages, acute form and with longer disease duration. In our patient the atypical EEG pattern was associated with asymmetric myoclonus, rapid evolution of weakness, motor aphasia and patient succumbing over a three month period.

\section{Pathogenesis}

The exact origin of GPC is uncertain. The development of functional electrotonic synapses may be a common feature underlying periodic EEG activity ${ }^{10}$. Measles virus is capable of inducing cell fusion in vitro and in vivo. The electrophysiological effects of virus induced cell fusion may include tight electrical coupling. Injured neurons do remain viable and even become abnormally excitable. One possible consequence of electrical coupling would be to permit the rapid and efficient electrotonic spread of paroxysmal burst discharges from cell to cell thus facilitating synchronous bursting within a large pool of neurons. 


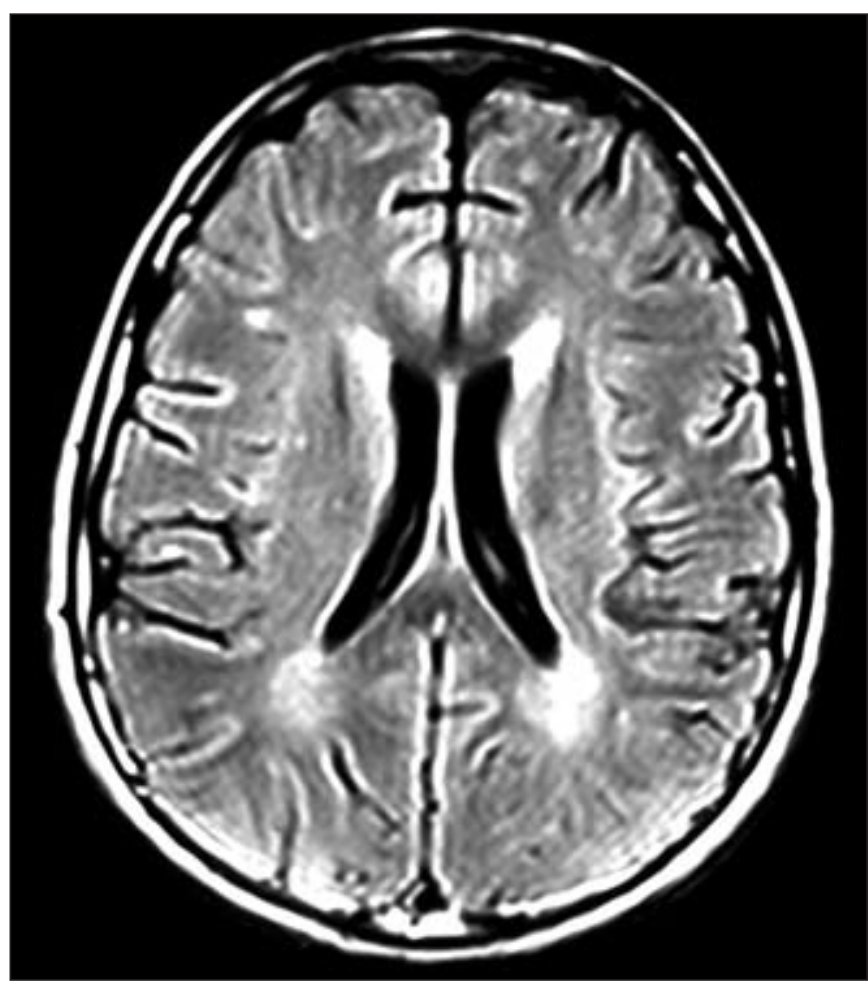

Figure 2: MRI brain (1.5 Tesla) fluid attenuated inversion recovery images showed bilateral peritrigonal hyperintensities.

The EEG correlate of this activity would be epileptiform discharges. Periodic synchronous bursting will occur if a burst in one cell is capable of inducing bursting in neighbouring cells ${ }^{10}$. Relative integrity of the cortex is critical for the appearance of these complexes. This is the reason proposed for the occurrence of GPC and a gradual waning in amplitude of complexes eventually resulting in an almost isoelectric record in the preterminal stages. Our subject's first EEG (Figure 1A) was taken early in the course of illness and the generalized discharges are well demonstrated.

Focal discharges in EEG are an uncommon finding in a global encephalitis like SSPE ${ }^{8}$. Hence presence of PLEDs at some stage of the disease process in SSPE would suggest a preferential involvement of one hemisphere. In the absence of a structural abnormality responsible for the occurrence of PLEDs, it seems likely that the electrophysiological change is confined to one hemisphere or manifests only in one hemisphere. Cellular mechanisms secondary to viral proliferation along with altered host immune responses aggravate the cortical neuronal injury. This associated with the adjacent white matter damage leads to a state of acute deafferentation of the cortex leading to the formation of PLEDs ${ }^{10,11}$, which are usually transitory and selflimiting, but can also be permanent ${ }^{12}$.

In conclusion, we report a case of SSPE with PLEDs, which has rarely been reported. It may signify a rapid fatal clinical outcome with focal symptoms during the disease course suggesting a preferential affliction of one hemisphere at some stage of the illness.

\section{REFERENCES}

1. Dyken PR. Subacute sclerosing panecephalitis. Neurol Clin. 1985; 3:179-95.

2. Markand ON, Panszi JG. The electroencephalogram in subacute sclerosisng panencephalitis. Arch Neurol. 1975;32:719-26.

3. Dogulu CF, Ciger A, Saygi S, Renda Y, Yalaz K. Atypical EEG findings in subacute sclerosing panencephalitis. Clin Electroencephalogr. 1995;26:193-9.

4. Gürses C, Oztürk A, Baykan B, Gökyiğit A, Eraksoy M, Barlas M, et al. Correlation between clinical stages and EEG findings of subacute sclerosing panencephalitis. Clin Electroencephalogr. 2000;31:201-6.

5. Silva DF, Lima MM, Anghinah R, Zanoteli E, Lima JG. Atypical clinical and electroencephalographic pattern in a patient with subacute sclerosing panencephalitis. Arq Neuropsiquiatr. 1995; 53:278-80.

6. Praveen-kumar S, Sinha S, Taly AB, Jayasree S, Ravi V, Vijayan J, et al. Electroencephalographic and imaging profile in a subacute sclerosing panencephalitis (SSPE) cohort: a correlative study. Clin Neurophysiol. 2007;118(9):1947-54.

7. García-Morales I, García MT, Galán-Dávila L, Gómez-Escalonilla C, Saiz-Díaz R, Martínez-Salio A, et al. Periodic lateralized epileptiform discharges: etiology, clinical aspects, seizures, and evolution in 130 patients. J Clin Neurophysiol. 2002;19(2): 172-7.

8. Shivji ZM, Al-Zahrani IS, Al-Said YA, Jan MM. Subacute sclerosing panencephalitis presenting with unilateral periodic myoclonic jerks. Can J Neurol Sci. 2003;30(4):384-7.

9. Ekmekci O, Karasoy H, Gokcay A, Ulku A. Atypical EEG findings in subacute sclerosing panencephalitis. Clin Neurophysiol. 2005; 116:1762-7.

10. Traub RD, Pedley TA. Virus-induced electronic coupling: hypothesis on the mechanism of periodic EEG discharges in Creutzfeldt-Jakob disease. Ann Neurol. 1981;10:405-10.

11. PeBenito R, Cracco JB. Periodic lateralized epileptiform discharges in infants and children. Ann Neurol. 1979;6:47-50.

12. Westmoreland BF, Klass DW, Sharbrough FW. Chronic periodic lateralized epileptiform discharges. Arch Neurol. 1986;43:494-6. 